Shusheng Fu, Department of Mathematics, University of Fuzhou, Fuzhou Fujian, P. R. China 350002

\title{
THE $s$-DIMENSIONAL HAUSDORFF INTEGRAL AND ITS PHYSICAL INTERPRETATION
}

\begin{abstract}
A relationship between the $s$-dimensional Hausdorff integral and the evolution with losses is established. Professor R. R. Nigmatullin showed that the evolution with loss can be described by a non-integer integral. This paper gives another way to describe the evolution. That is, the evolution can be expressed as Hausdorff integral.
\end{abstract}

\section{Introduction}

The Lebesgue integral is the most important integration theory in mathematics and natural science. However the Lebesgue theory can not deal with the properties of functions defined on a set $E$ of Lebesgue measure $m E=0$. The Hausdorff Calculus studies the differentiation and integration of functions defined on a set of Lebesgue measure zero. Since many properties of functions depend on the behavior of functions on a set of Lebesgue measure zero, one should not be surprised that the Hausdorff calculus is applicable in these cases. Using these newly defined concepts, the Cantor ternary function is an indefinite integral [FU1] and length of this kind of singular curve has an integral representation [FU2], both results can not be achieved by Lebesgue Theory.

The purpose of this paper is to give a physical interpretation to the Hausdorff integral. In his pioneer work [N1], Professor R. R. Nigmatullin studies

Key Words: net Hausdorff derivative, net Hausdorff integral, singular function, evolution with losses

Mathematical Reviews subject classification: Primary: 26A39, 51M25; Secondary: $28 \mathrm{~A} 75$

Received by the editors October 12, 1994 
the evolution of some physical systems with losses using non-integer integrals. In this paper it is shown that such evolution can be exactly described by the Hausdorff integral. First, let us quote the nice description from Professor Nigmatullin's letter "let us suppose that an initial bar made from metal has the length $t$ and density $P_{0}=1$. That initial element is divided in two parts of length $\xi t$ but each bar becomes heavier in the result of reforming and has the mass $\frac{1}{2 \xi t}$. This procedure keeps the total mass of the initial bar". The procedure continues infinitely and gives a curve $M(t)$ which described the distribution of masses on the chosen "length" $t$. As a result the mass concentrates in an "exception" set (Lebesgue measure zero) and there is no mass for almost every point in interval $[0, t]$. In other words, the density of mass is 0 for almost every point, but it becomes infinite on an uncountable set of Lebesgue measure zero. The traditional analysis can not deal with this phenomenon. What Professor Nigmatullin did is that he approximated the loss by the sum $P_{n}(t)=\frac{1}{(2 \xi)^{n} t} \int_{0}^{t} d \tau \sum_{m=1}^{2^{n}} \eta\left(t_{m}^{(n)}<\tau<t_{m+1}^{(n)}\right) f(\tau)$, the total loss $P(t)$ is then defined by $P(t)=\lim _{n \rightarrow \infty} P_{n}(t)$. The following shows the relationship between the loss $P(t)$ and the Hausdorff integral.

The Hausdorff integral is a Riemann type integral. It is defined as the limit of Riemann sum $S_{D}=(D) \sum f(\tau) H^{s}(E \cap[u, v])$, where $s=\log 2 / \log \frac{1}{\xi}$ denotes the Hausdorff dimension of Cantor set $E$. Note that

$$
S_{n}=\sum_{m=1}^{2^{n}} f\left(\tau_{m}\right) H^{s}\left(E \cap\left[u_{m}, v_{m}\right]\right)=\sum_{m=1}^{2^{n}} f\left(\tau_{m}\right) \frac{t^{s}}{2^{n}}
$$

and

$$
\begin{aligned}
P_{n}(t) & =\frac{1}{(2 \xi)^{n} t} \int_{0}^{t} d \tau \sum_{m=1}^{2^{n}} \eta\left(t_{m}^{(n)}<\tau<t_{m+1}^{(n)}\right) f(\tau) \\
& =\sum_{m=1}^{2^{n}} \frac{\tau_{m}}{(2 \xi)^{n} t} \xi^{n} t=\sum_{m=1}^{2^{n}} f\left(\tau_{m}\right) \frac{1}{2^{n}}
\end{aligned}
$$

then we have $P(t)=t^{-s}(H) \int_{0}^{t} f(\tau) d H^{s}$. Recently, Professor Nigmatullin asked the author if I can get these results [N1] without using Laplace transform (private communication). This paper can be considered as an answer to Professor Nigmutallin's problem.

\section{Notations and Terminology}

Let $[a, b]$ be a closed bounded interval and let $E$ be a perfect set of Lebesgue measure $m E=0, H-\operatorname{dim} E=s \quad(0<s<1)$ and $0<H^{s}(E)<\infty$, where 
$H$-dim denotes the Hausdorff dimension, the $H^{s}$ Hausdorff measure. Following the terminology of B. B. Mandelbrot [MA, p. 74] such a set $E$ is called a perfect dust. Set $[a, b] \backslash E=\bigcup\left(a_{i}, b_{i}\right)$ and let $b_{i_{0}}-a_{i_{0}}=\max \left\{b_{i}-a_{i}: i=1,2,3, \ldots\right\}$, define $E_{1}^{1}=\left[\alpha_{1}^{1}, \beta_{1}^{1}\right]=\left[a, a_{i_{0}}\right], E_{1}^{2}=\left[\alpha_{1}^{2}, \beta_{1}^{2}\right]=\left[b, b_{i_{0}}\right]$. Let $b_{i_{1}}-a_{i_{1}}=$ $\max \left\{b_{i}-a_{i}:\left[a_{i}, b_{i}\right] \subset\left[\alpha_{1}^{1}, \beta_{1}^{1}\right]\right\}$ and $b_{i_{2}}-a_{i_{2}}=\max \left\{b_{i}-a_{i}:\left[a_{i}, b_{i}\right] \subset\left[\alpha_{1}^{2}, \beta_{1}^{2}\right]\right\}$. Define $E_{2}^{1}=\left[\alpha_{2}^{1}, \beta_{2}^{1}\right]=\left[a, a_{i_{1}}\right], \ldots, E_{2}^{4}=\left[\alpha_{2}^{4}, \beta_{2}^{4}\right]=\left[b_{i_{2}}, b\right]$. This procedure continues to give a sequence of intervals, $\left\{\left[\alpha_{n}^{i}, \beta_{n}^{i}\right]_{i=1}^{2^{n}}\right\}, n=1,2, \ldots$ Let $E_{n}=\bigcup_{i=1}^{2^{n}} E_{n}^{i}, n=1,2, \ldots$ then $E=\bigcap_{n=1}^{\infty} E_{n}$. It is clear that the class $\left\{E_{n}^{i}\right\}_{i=1}^{2^{n}}, n=1,2, \ldots$, forms a net of sets [FA, p. 64].

The upper and lower net densities of $E$ at a point $x \in E \backslash \bigcup\left\{a_{i}, b_{i}\right\}$ are defined as

$$
\begin{aligned}
& \overline{N D}(E, x)=\varlimsup_{n \rightarrow \infty} \frac{H^{s}\left(E \cap\left[\alpha_{n}^{i_{x}}, \beta_{n}^{i_{x}}\right]\right)}{\left(\beta_{n}^{i_{x}}-\alpha_{n}^{i_{x}}\right)^{s}} \\
& \underline{N D}(E, x)=\lim _{n \rightarrow \infty} \frac{H^{s}\left(E \cap\left[\alpha_{n}^{i_{x}}, \beta_{n}^{i_{x}}\right]\right)}{\left(\beta_{n}^{i_{x}}-\alpha_{n}^{i_{x}}\right)^{s}}
\end{aligned} \quad x \in\left(\alpha_{n}^{i_{x}}, \beta_{n}^{i_{x}}\right), \quad n=1,2, \ldots
$$

respectively. If $\overline{N D}(E, x)=\underline{N D}(E, x)$ we say that the net density of $E$ at $x$ exists, and we write $N D(E, x)$ for the above value. A dust $E$ is said to be normal if there is a positive number $\beta$ such that $\underline{N D}(E, x) \geq \beta H$-a.e.

Remark 1 The ordinary density is not suitable for doing calculus. It can happen that $\underline{D}(E, x)=0$ for all $x$, even though $H^{s}(E)>0$ [FE, p. 294]. Moreover, it is hard to estimate the lower density $\underline{D}(E, x)$. However, it can be shown that net density is 1 at the points of a Cantor set $C$ nearly everywhere (except on a countable set). Consequently, the Cantor set is normal. This is why we use the net density instead of ordinary density.

For any singular function on $E$, we define the net extreme Hausdorff derivatives as

$$
\begin{aligned}
& \overline{N-H D} F(x)=\varlimsup_{n \rightarrow \infty} \frac{F\left(\beta_{n}^{i_{x}}\right)-F\left(\alpha_{n}^{i_{x}}\right)}{H^{s}\left(E \cap\left[\alpha_{n}^{i_{x}}, \beta_{n}^{i_{x}}\right)\right.} \\
& \underline{N-H D} F(x)=\lim _{n \rightarrow \infty} \frac{F\left(\beta_{n}^{i_{x}}\right)-F\left(\alpha_{n}^{i_{x}}\right)}{H^{s}\left(E \cap\left[\alpha_{n}^{i_{x}}, \beta_{n}^{i_{x}}\right)\right.} \\
& x \in\left(\alpha_{n}^{i_{x}}, \beta_{n}^{i_{x}}\right), \quad n=1,2, \ldots
\end{aligned}
$$

If $\overline{N-H D} F(x)=N-H D F(x)$, we say that the function $F(x)$ is net Hausdorff differentiable at $x$; this limit is termed the net Hausdorff derivative of $F(x)$ at $x$ and is denoted by $F_{S H}^{\prime}(x)$.

Remark 2 If the dust $E$ is a Cantor type set, then the net Hausdorff derivative coincides with $\lambda$-power dyadic derivative [FU1]. 
We need some notations from Henstock integration theory. Let $I$ be the set of all closed intervals $[u, v]$ in $[a, b]$ having a nonempty interior. An element $([u, v], x) \in I \times[a, b]$ is called an interval-point pair. We write $D=\{([u, v], x)\}$ where $([u, v], x)$ denotes a typical interval-point pair in $D$. Then $D$ is said to be a partition of $[a, b]$ (E resp.) if $\{[u, v] ;([u, v], x) \in D\}$ is a finite collection of nonoverlapping subintervals of $[a, b]$ and $[a, b]=\bigcup_{([u, v], x) \in D}[u, v](E=$ $\left.\bigcup_{([u, v], x) \in D}[u, v] \cap E\right)$. Let $\delta(x)$ be a positive function on $[a, b](E)$. The partition $D$ is called a $\delta$-fine division of $[a, b](E)$ if $([u, v], x) \in D$, then $v-u \leq \delta(x)$. A $\delta$-fine division $D$ of $[a, b]$ is called a net $\delta$-fine division of $[a, b]$, if $E=\bigcup_{([u, v], x) \in D}[u, v] \cap E$ and $[u, v]=\left[\alpha_{n}^{i_{x}}, \beta_{n}^{i_{x}}\right]$, for $x \in E$. Thomson's paper $[\mathrm{TH}]$ provides a nice introduction to these terminologies.

\section{$3 s$-dimensional Hausdorff integral}

As it was mentioned in the introduction, there are some physical systems in which the mass concentrates in a set $E$ of Lebesgue measure $m E=0$. Therefore, the integral which sums up the total mass will take place only on $E$.

Definition 1 A real function $f$ on $E$ is said to be Hausdorff integrable on $[a, b]$, if there exists $r$, such that for every $\epsilon>0$ there is a gauge function $\delta(x)>0$ on $E$, such that for any net $\delta$-fine division $D=\{([u, v], x)\}$ of $E$, we have

$$
\left|(D) \sum f(x) H^{s}(E \cap[u, v])-r\right|<\epsilon,
$$

As usual, we write

$$
\text { (H) } \int_{a}^{b} f(x) d H^{s}=r .
$$

For $x \in[a, b]$, let $F(x)=(H) \int_{a}^{x} f(x) d H^{s}+F(a)$. We call $F(x)$ an indefinite Hausdorff-integral on $[a, b]$.

Lemma 1 (Covering Lemma) Let $\delta(x)>0$ for $x \in E$, then there is a net $\delta$-fine division of $E$.

Proof. Let $F=\{x \in E: x$ is an endpoint of a complementary interval of $E\}$. For each $x \in E \backslash F$, there is a positive integer $N_{x}$ such that $x \in\left(\alpha_{n}^{i_{x}}, \beta_{n}^{i_{x}}\right) \subset$ $(x-\delta(x), x+\delta(x))$ whenever $n \geq N_{x}$. For each $x \in F$, i.e., $x=\alpha_{n}^{i_{x}}$ or $\beta_{n}^{i_{x}}$ for some $n$, there is an element $E_{m_{x}}^{i_{m_{x}}}$ of net $\left\{E_{m_{x}}^{i}\right\}^{\alpha^{i_{x}}}$ such that $I_{x}$ one-sided intersects with $E, I_{x}=\left(2 \alpha_{n}^{i_{x}}-\beta_{m_{x}}^{i_{m_{x}}}, \beta_{m_{x}}^{i_{m_{x}}}\right)$ or $I_{x}=\left(\alpha_{m_{x}}^{i_{m_{x}}}, 2 \beta_{n}^{i_{x}}-\alpha_{m_{x}}^{i_{m_{x}}}\right)$ and $\left|E_{m_{x}}^{i_{m_{x}}}\right|<\delta(x)$. Since $E$ is compact and $E \subset \cup\left\{I_{x}: I_{x}=\left(\alpha_{n_{x}}^{i_{n_{x}}}, \beta_{n_{x}}^{i_{n_{x}}}\right)\right.$, whenever 
$x \in E \backslash F ; I_{x}=\left(2 \alpha_{n_{x}}^{i_{n_{x}}}-\beta_{m_{x}}^{i_{m_{x}}}, \beta_{m_{x}}^{i_{m_{x}}}\right)$, or $\left(\alpha_{m_{x}}^{i_{m_{x}}}, 2 \beta_{n_{x}}^{i_{n_{x}}}-\alpha_{m_{x}}^{i_{m_{x}}}\right)$, whenever $\left.x \in F\right\}$; then by Heine-Borel theorem there is a finite subcover $A=\left\{I_{x_{i}}\right\}_{i=1}^{I}$ of $E$. We divide the intervals $I_{x_{i}}, i=1,2, \ldots, I$, into two groups, we shall say that an interval $I_{x_{i}}$ is of the the first class if the associated point $x_{i} \in E \backslash F$; otherwise the second class i.e., the associated point $x_{i} \in F$.

Since we are interested in a net $\delta$-fine division of $E$, we shall show that with a suitable extension of $\delta$ to all $[a, b]$, we can construct a net $\delta$-fine division of $[a, b]$. This will give the required result. We show that there is a net $\delta$-fine division of $[a, b]$. First, we mention a property of the net which will be used in following. Let $\left[\alpha_{m}^{i}, \beta_{m}^{i}\right],\left[\alpha_{p}^{j}, \beta_{p}^{j}\right]$ be the elements of net $\left\{E_{n}^{i}\right\}_{i=1}^{2^{n}}$, $n=1,2, \ldots$, then either $\left(\alpha_{m}^{i}, \beta_{m}^{i}\right) \cap\left(\alpha_{p}^{j}, \beta_{p}^{j}\right)=\emptyset$ or $\left(\alpha_{m}^{i}, \beta_{m}^{i}\right) \subseteq\left(\alpha_{p}^{j}, \beta_{p}^{j}\right)$ or $\left(\alpha_{p}^{j}, \beta_{p}^{j}\right) \subseteq\left(\alpha_{m}^{i}, \beta_{m}^{i}\right)$. Let $U=\bigcup_{i=1}^{I} I_{x^{i}}=\bigcup_{p=1}^{P}\left(u_{p}, v_{p}\right)$, where open intervals $\left(u_{p}, v_{p}\right)$ are pairwise disjoint. Note that for each $p=1,2, \ldots, P,\left(u_{p}, v_{p}\right)$ is of finite union of elements of $A$, that is, $\left(u_{p}, v_{p}\right)=\bigcup_{t=1}^{T} I_{x^{i} t}, I_{x^{i} t} \in A$. If necessary discard some intervals $I_{x^{i} t}$, we write $I_{x^{i} t}=\left(c_{t}, d_{t}\right), t=1,2, \ldots, T$, such that

$$
u_{j}=c_{1}<c_{2}<d_{1}<c_{3}<d_{2}<\cdots<d_{T}=v_{j} .
$$

Since $E$ is covered by $A$, there are three possible cases of $\left(c_{1}, d_{1}\right)$. We treat one case only. We may write

$$
\left(c_{1}, d_{1}\right)=\left(c_{1}, \alpha_{m_{1}}^{i_{1}}\right) \cup\left[\alpha_{m_{1}}^{i_{1}}, \beta_{m_{1}}^{i_{1}}\right)
$$

For the first class interval $\left(c_{2}, d_{2}\right)$, by the property of the net we must have

$$
\left[\alpha_{m_{1}}^{i_{1}}, \beta_{m_{1}}^{i_{1}}\right] \subseteq\left[c_{2}, d_{2}\right]=\left[\alpha_{m_{2}}^{i_{2}}, \beta_{m_{2}}^{i_{2}}\right]
$$

where $\alpha_{m_{1}}^{i_{1}}=\alpha_{m_{2}}^{i_{2}}$ then,

$$
\left(c_{1}, d_{2}\right)=\left(c_{1}, \alpha_{m_{2}}^{i_{2}}\right) \cup\left[\alpha_{m_{2}}^{i_{2}}, \beta_{m_{2}}^{i_{2}}\right) .
$$

For the second class interval $\left(c_{2}, d_{2}\right)$, the interval $\left(c_{2}, d_{2}\right)$ intersects with $E$ on the left-side. Therefore, by the property of the net, we may write

$$
\left(c_{1}, d_{2}\right)=\left(c_{1}, \alpha_{m_{1}}^{i_{1}}\right) \cup\left[\alpha_{m_{1}}^{i_{1}}, \beta_{m_{1}}^{i_{1}}\right] \cup\left(\beta_{m_{1}}^{i_{1}}, d_{2}\right)
$$

or

$$
\left(c_{1}, d_{2}\right)=\left(c_{1}, \alpha_{m_{2}}^{i_{2}}\right) \cup\left[\alpha_{m_{2}}^{i_{2}}, \beta_{m_{2}}^{i_{2}}\right] \cup\left(\beta_{m_{2}}^{i_{2}}, d_{2}\right) .
$$

Similarly for the other cases of $\left(c_{1}, d_{1}\right)$. Using the definition of $\alpha_{m}^{j}$ and $\beta_{m}^{j}$ and the above results, one may choose the elements from $A$ so that the union of the first class intervals and the first class subintervals of the second class intervals form a partial partition of $E \cap\left(c_{1}, d_{2}\right)$. The procedure continues and 
stops in finite steps. Thus, there exists a partition $D_{1}$ of $E$ which consists of the first class intervals and the first class subintervals of the second class intervals.

Since $D_{1}$ is a finite collection of subintervals of $[a, b]$, then $V=[a, b] \backslash U, U=$ $\bigcup_{[u, v] \in D_{1}}[u, v]$, is a finite collection of subintervals of $[a, b]$. Define a gauge function $\delta_{1}(x)$ on each closed interval of $V$ by

$$
\delta_{1}(x)= \begin{cases}\delta(x) & x \text { is an endpoint of intervals of } V \\ \frac{1}{2} d(x, U) & x \in V .\end{cases}
$$

It is well known that for every positive function $\eta(x)$ defined on an interval $[\alpha, \beta]$. There is a $\eta$-fine division $D$ on $[\alpha, \beta]$. Applying this statement to each closed interval of $V$, we get a partition $D_{2}$ of $V$. We write $D=D_{1} \cup D_{2}$, then $D$ is a net $\delta$-fine division of $[a, b]$.

By the Lemma, the $s$-dimensional Hausdorff integral is well defined. The usual properties of ordinary integral hold for the $s$-dimensional Hausdorff integral.

\section{Physical Interpretation}

We first recall the construction of Professor Nigmatullin. It is known that the Cantor set is constructed by the following algorithm. Begin with taking $t$ as the total length of a bar of weight 1 . In step one, we remove the middle open subinterval, leaving two subintervals with length $\xi t\left(\xi<\frac{1}{2}\right)$. In step two, the same procedure applies to the remaining subintervals with length $\xi t$. Then the procedure continues infinitely (see Figure 1). After the first partition the remaining intervals are $[0, \xi t],[t(1-\xi), t]$; the inserted points are $\xi t, t(1-\xi)$, and the density of the remaining points is $(2 \xi t)^{-1}$. After the second partition the remaining intervals are $\left[0, \xi^{2} t\right],\left[\left(\xi-\xi^{2}\right) t, \xi t\right],\left[t(1-\xi), t\left(1-\xi+\xi^{2}\right)\right]$ and $\left[t\left(1-\xi^{2}\right), t\right]$, the inserted points are $\xi^{2} t,\left(\xi-\xi^{2}\right) t, t(1-\xi),\left(1-\xi+\xi^{2}\right) t, t\left(1-\xi^{2}\right)$, the density of the remaining points is $\frac{1}{(2 \xi)^{2} t}$. Let $t_{m}^{(n)}\left(m=1,2, \ldots, 2^{n}\right)$ denote the coordinates of the $n$-step inserted points then the coordinates of the $(n+1)$ step inserted points are defined by following recurrence relationship

$$
\begin{array}{ll}
t_{m+1}^{(n+1)}=t_{m}^{(n)}, & t_{m+2}^{(n+1)}=t_{m}^{(n)}+\xi^{n+1} t \\
t_{m+3}^{(n+1)}=t_{m+1}^{(n)}-\xi^{n+1} t, & t_{m+4}^{(n+1)}=t_{m+1}^{(n)},
\end{array}
$$

with the density $\frac{1}{(2 \xi)^{n+1} t}$. 


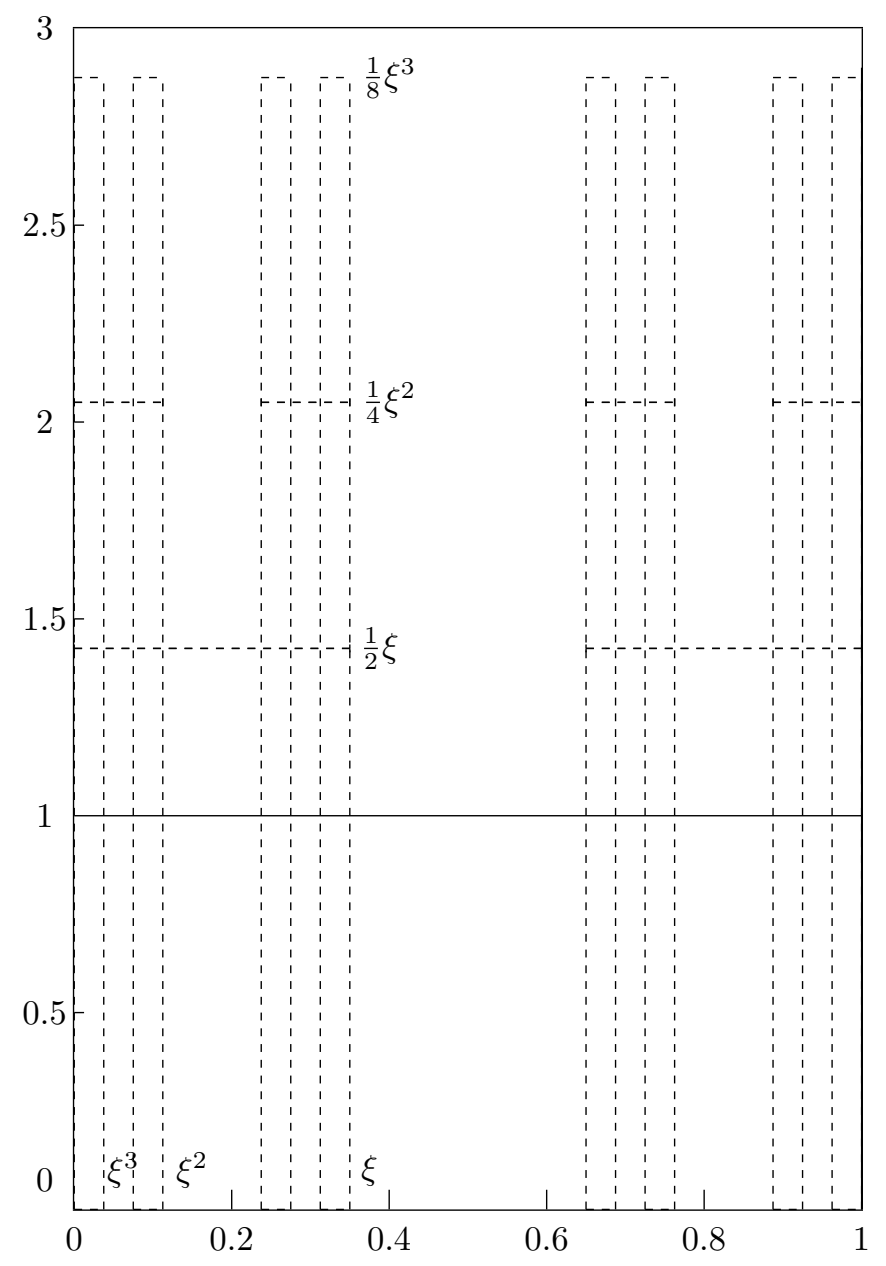

Figure 1.

We now integrate $f(\tau)$ over the $2^{n}$ small strips $\left[t_{2 m-1}^{(n)}, t_{2 m}^{(n)}\right]$, obtaining

$$
P_{n}(t)=\frac{1}{(2 \xi)^{n} t} \int_{0}^{t} d \tau \sum_{m=1}^{2^{n}} \eta\left(t_{2 m-1}^{(n)}<\tau<t_{2 m}^{(n)}\right) f(\tau)
$$

We define

$$
P(t)=\lim _{n \rightarrow \infty} P_{n}(t)
$$


Theorem 1 Let $f$ be a continuous function on $E$, then

$$
P(t)=t^{-s}(H) \int_{0}^{t} f(\tau) d H^{s} .
$$

Proof. It is clear that the Hausdorff integral $(H) \int_{0}^{t} f(\tau) d H^{s}$ exists for every continuous function $f(\tau)$ on $E$. We want to show $\lim _{n \rightarrow \infty} P_{n}(t)=$ $t^{-s}(H) \int_{0}^{t} f(\tau) d H^{s}$.

Given $\epsilon>0$, there is a positive number $\delta$, such that

$$
\left|f(\tau)-f\left(\tau^{\prime}\right)\right|<\epsilon
$$

whenever $\left|\tau-\tau^{\prime}\right|<\delta$. We can find a positive integer $N$, such that $\xi^{n} t<\delta$, so that

$$
(f(\tau)-\epsilon) \frac{\xi^{n} t}{(2 \xi)^{n} t}<\frac{1}{(2 \xi)^{n} t} \int_{t_{2 m}^{(n)}}^{t_{2 m-1}^{(n)}} f(\tau) d \tau<(f(\tau)+\epsilon) \frac{\xi^{n} t}{(2 \xi)^{n} t}
$$

where $t_{2 m-1}^{(n)} \leq \tau \leq t_{2 m}^{(n)}$ and $n \geq N$. Thus,

$$
\left|P_{n}(t)-\sum_{1}^{2^{n}} f\left(\tau_{m}\right) \frac{1}{2^{n}}\right|<\epsilon
$$

whenever $n \geq N$ and $\tau_{m} \in\left[t_{2 m-1}^{(n)}, t_{2 m}^{(n)}\right]$.

By the definition of Hausdorff integral, there is a gauge function $\delta_{1}(x)$, such that

$$
\left|\left(D_{1}\right) \sum f(\tau) H^{s}(E \cap[u, v])-(H) \int_{0}^{t} f(\tau) d H^{s}\right|<\epsilon
$$

for every net $\delta_{1}$-fine division $D$ of $E$. Let $\delta_{2}(x)=\min \left(\delta_{1}(x), \delta\right)$, by Lemma 1 there is a net $\delta_{2}$-fine devision $D_{1}$, note that

$$
\left(D_{1}\right) \sum f(\tau) H^{s}(E \cap[u, v])=\sum_{1}^{2^{n}} f\left(\tau_{m}\right) \frac{t^{s}}{2^{n}}
$$

where $\tau_{m} \in\left[t_{2 m-1}^{(n)}, t_{2 m}^{(n)}\right]$ for some $n$.

Combining (2) and (3) gives

$$
\left|P_{n}(t)-t^{-s}(H) \int_{0}^{t} f(\tau) d H^{s}\right|<\left(1+t^{-s}\right) \epsilon,
$$


and so $\lim _{n \rightarrow \infty} P_{n}(t)=t^{-s}(H) \int_{0}^{t} f(\tau) d H^{s}$.

Acknowledgments. The author wishes to thank Prof R. Nigmatullin for his helpful comments. Special thanks to Prof B. Thomson and the referee for their constructive suggestions.

\section{References}

[FA] K. J. Falconer, The geometry of fractal Sets, Cambridge Univ. Press, London and New York, 1985.

[FE] H. Federer, Colloquium Lectures on geometric Measure theory, Bull. Amer. Math. Soc. 84, 1978.

[FU1] S. S. Fu, $\lambda$-power integrals on the Cantor type sets, to appear in Proc. Amer. Math. Soc..

[FU2] S. S. Fu, Hausdorff Calculus and Arc Length, submitted.

[MA] B. B. Mandelbrot, The fractal geometry of nature, San Francisco, W. H. Freeman \& Co., 1982.

[N1] R. R. Nigmatullin, A fractional integral and its physical interpretation, Teoret. Met. Fiz, 90 no. 3 (1992), 354-368; Translation in Theoret. and Math. Phy, 90 no. 3 (1992), 242-251.

$[\mathrm{TH}]$ B. S. Thomson, Differentiation bases on the real line, I and II, Real Analysis Exchange, 7 (1982-83), 67-208; 8 (1982-83) 278-442. 